\title{
BORDE EN CONFLICTO, CASO DE ESTUDIO: LOMAS DE AMANCAES
}

\author{
EDGE IN CONFLICT, CASE STUDY: \\ LOMAS DE AMANCAES
}

XIMENA SUÁREZ

Universidad de Lima

Las lomas son ambientes naturales de condiciones ecológicas especiales, que surgen del encuentro de estribaciones costeras y la neblina invernal. Su vasta riqueza natural, con flora y fauna endémica, es exclusiva del Perú y parte de Chile. Sin embargo, este ecosistema se ha visto seriamente descuidado por el Estado, a pesar de contar con grandes beneficios para la población. Actualmente, las lomas de Amancaes atraviesan su mayor reto, el de subsistir frente a una fuerte presión urbana que no parece tener intenciones de detenerse; por ello, urge la presencia de una intervención que regule el conflicto en el borde natural-urbano.

borde natural-urbano, borde en conflicto, lomas costeras, lomas de Amancaes, arquitectura de

\section{RODRIGO TORNERO}

Universidad de Lima

Recibido: 15 de agosto del 2020

Aprobado: 3 de noviembre del 2020

doi: https://doi.org/10.26439/limaq2021.n008.5556

Coastal hills are natural environments with special ecological conditions that arise from coastal foothills and winter fog. Their vast natural wealth, with endemic flora and fauna, is exclusive to Peru and part of Chile. However, the State has seriously neglected this ecosystem, despite having significant benefits for the population. Currently, the Amancaes coastal hills are going through their most significant challenge, that of surviving in the face of strong urban pressure that does not seem to have any intention of stopping; therefore, the presence of an intervention that regulates the conflict on the natural-urban edge is urgent.

urban-natural edge, edge in conflict, coastal knolls, Amancaes knolls, architecture edge 


\section{INTRODUCCIÓN}

Este artículo subraya la necesidad de un proyecto pertinente que responda a las necesidades de la población y promueva la conservación de las lomas de Amancaes. Además, se destaca la particularidad de intervenir un borde en permanente conflicto socioeconómico, cuya solución se alinea con la necesidad de preservar el ecosistema para los futuros habitantes de Amancaes y de Lima Metropolitana.

Para la obtención de la información se consultaron fuentes primarias como entrevistas, visitas de campo, dinámicas participativas con la población y datos cuantitativos que se obtuvieron del Instituto de Estadística e Informática (INEI). También se recurrió a fuentes secundarias, tales como tesis de titulación en arquitectura, artículos científicos de revistas indexadas e informes nacionales oficiales.

\section{LAS LOMAS COMO BORDES URBANOS}

Cuando visitamos un lugar por primera vez, por lo general, no somos conscientes del valor intrínseco que este posee. Una visión superficial nos limita a percibir el territorio con una incorrecta abstracción del imaginario colectivo $^{1}$ y la memoria del lugar. Este enfoque nos conduce a una interpretación prejuiciosa de los territorios ajenos a nuestra realidad cotidiana, la cual genera una barrera entre el usuario y la sensibilización espacial.

Si extrapolamos esta idea a la realidad urbana limeña, al momento de representar los escenarios del centro y la periferia de la ciudad, se evidencia la ignorancia de la cualidad dinámica del borde, como un lugar cargado de memoria, escenas, vivencias y elementos de identidad cultural, centrando nuestra atención solo en la precariedad y carencia material.

Según Lynch (1959), un borde se concibe como un límite de la ciudad que separa espacios diferenciados socialmente: lo natural y lo construido. Es decir, se puede entender como una transición entre lo urbano y lo natural, producto del crecimiento hacia el exterior de la ciudad. Este panorama es constante en Latinoamérica, donde al haberse ocupado las periferias llanas, se inicia el asentamiento en accidentes geográficos del territorio, tales como las laderas. Caballero Zeitún (2011) conceptualiza el término de ladera urbana como una manera de ocupar el suelo en pendientes de alta o moderada inclinación, con uso intensivo de vivienda, equipamiento urbano e infraestructura económica y social, donde se emplazan los asentamientos humanos.

Las principales preocupaciones de la ocupación de estas áreas son la pérdida tanto de tierras útiles para actividades agropecuarias como de reservas naturales

1 Cuando hablamos del imaginario colectivo, nos referimos a la síntesis de la representación que tenemos de la ciudad, la manera en la que observamos sus formas, usamos sus espacios y lo que esto significa para nosotros. Este concepto, adicionado a la cultura, conforma la memoria colectiva (Aguilera-Martínez et al., 2017). 
que sustentan la vida de las ciudades, y también la elevada inversión por parte del Estado para mitigar los impactos que genera dicho asentamiento (Toro Vasco, 2005). Esto se debe a que los ocupantes, que pertenecen a los estratos más bajos de la sociedad, tienen menos alternativas para elegir dónde vivir; por lo tanto, la necesidad básica de habitar prevalece frente al impulso de conservar un ambiente natural. Este es el caso de las invasiones en ecosistemas frágiles, tales como las lomas costeras. Dicho hábitat, según Nieuwland y Mamani (2017), se define como un oasis de neblina de alta biodiversidad de flora y fauna, en medio de un entorno árido formado por precipitaciones de las nieblas invernales.

Actualmente, un caso alarmante de asentamiento (véase la figura 1) en ladera en condiciones de depredación natural, olvido de la memoria histórica y un indebido sentimiento de apropiación es el que se desarrolla en las laderas de las lomas de Amancaes, en el distrito del Rímac, donde la dinámica presenta una serie de conflictos internos, que van desde la marginación y segregación de la población frente al resto de la ciudad, hasta el tráfico de terrenos en las laderas, que atenta contra el medio natural.

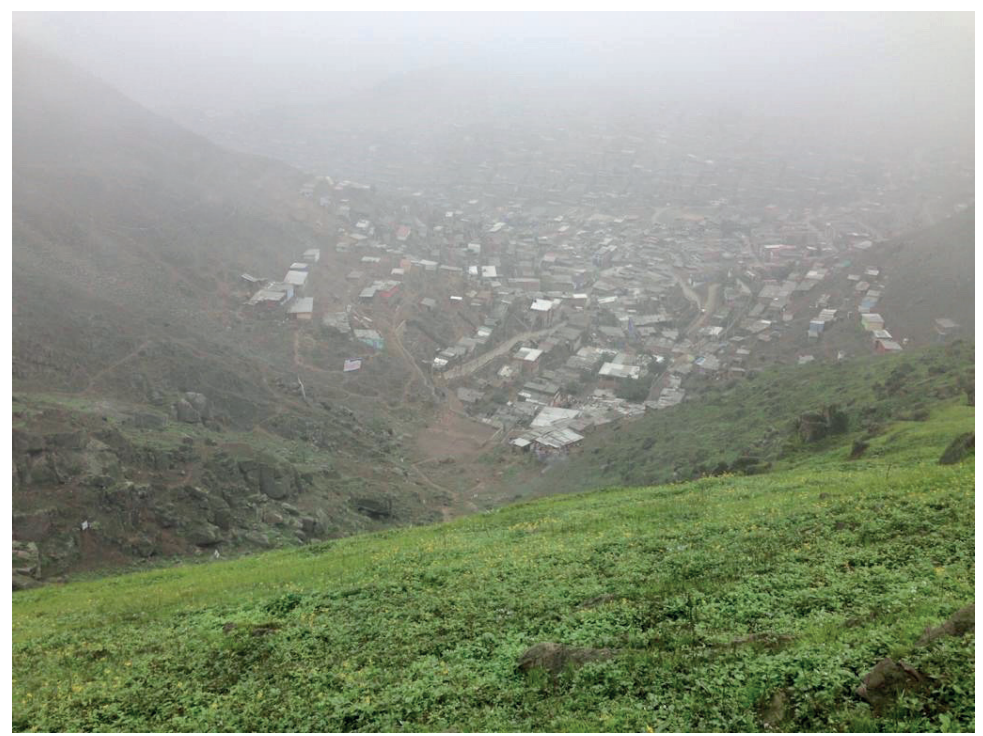

Según el Servicio Nacional de Áreas Naturales Protegidas (SERNANP, 2013), las lomas son ambientes naturales de condiciones ecológicas especiales que surgen del encuentro de estribaciones costeras y neblina invernal en la temporada de mayo a octubre. Su riqueza natural es vasta, con flora y fauna endémica, cuyo paisaje es exclusivo del Perú y parte de Chile. Estudios recientes del Instituto Metropolitano de Planificación (IMP) y el Servicio de Parques de Lima (SERPAR) registran 70000 hectáreas de lomas en el Perú, en las épocas más húmedas. En el ámbito limeño, según el Programa de las Naciones Unidas para el Desarrollo (PNUD, 2018), las lomas alcanzan una extensión que representa el $7 \%$ del territorio provincial, con casi 20000 
hectáreas. Actualmente, se encuentran en peligro de desaparecer, debido a la constante agresión de la mano del hombre. Pese a su biodiversidad y el valor que tienen para la ciudad, se ven obligadas a retroceder, a tal punto que su desaparición parece ser casi inevitable.

Este ecosistema otorga una gran cantidad de beneficios a la ciudad, como el incremento de la calidad del aire, sirve de refugio y fuente alimenticia para la fauna existente, es un espacio cercano a la ciudad para la educación científica y ambiental, mitiga los gases del efecto invernadero, capta agua atmosférica y contribuye a la formación del suelo (PNUD, 2018). Sin embargo, en el Perú, menos del $5 \%$ de la superficie de desiertos y matorrales xéricos está protegida por el Estado; es decir, este ecosistema se encuentra seriamente descuidado en ese ámbito. Uno de los casos más preocupantes es el de las lomas de Amancaes o San Jerónimo, que han sufrido una veloz degradación por parte del asentamiento humano Flor de Amancaes, en el distrito del Rímac; no obstante, a fines del 2019, se aprobó el Decreto Supremo 011-2019-MINAM, que establece el Área de Conservación Regional Sistema de Lomas de Lima (lomas de Ancón, Carabayllo, Amancaes y Villa María); esta medida servirá de respaldo para las propuestas tomadas con base en la recuperación de dichos ecosistemas.

Las lomas de Amancaes se encuentran entre los distritos de Independencia, San Juan de Lurigancho y, en su mayoría, en el Rímac, donde más del $25 \%$ del territorio comprende pendientes muy empinadas, que principalmente son las zonas de laderas del cerro San Jerónimo, es decir, el borde de las lomas de Amancaes, que mantiene una pendiente que va del $22,2 \%$ al 38,9 \%. El terreno es de carácter pedregoso y presenta peñascos (véase la figura 2), es decir, rocas de gran tamaño en estado natural, que inician a los $380 \mathrm{~m} \mathrm{~s}$. n. m. y llegan hasta los 750 m s. n. m. (Del Castillo, 2017).

Figura 2.

Vista de la boca de

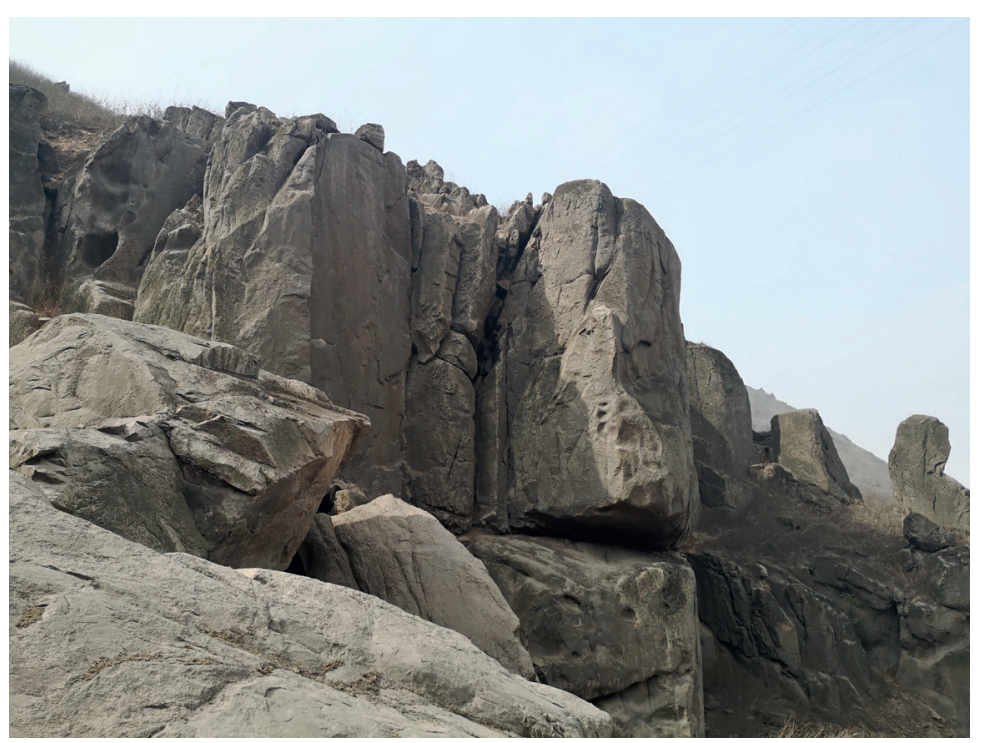


Una característica de las lomas es la niebla (véase la figura 3), que surge, en primer lugar, por la condensación del vapor de agua de la atmósfera y por la presencia de partículas higroscópicas (que son microscópicas), las cuales absorben la humedad del aire y la convierten en gotas al condensarla (Corell, 2014). Las condiciones climáticas de las lomas presentan un alto índice de humedad atmosférica y temperaturas bajas. La alta humedad relativa es el resultado de la colisión de la niebla contra la ladera de las lomas, y alcanza hasta índices del 94 \% (Municipalidad de Lima, 2019).

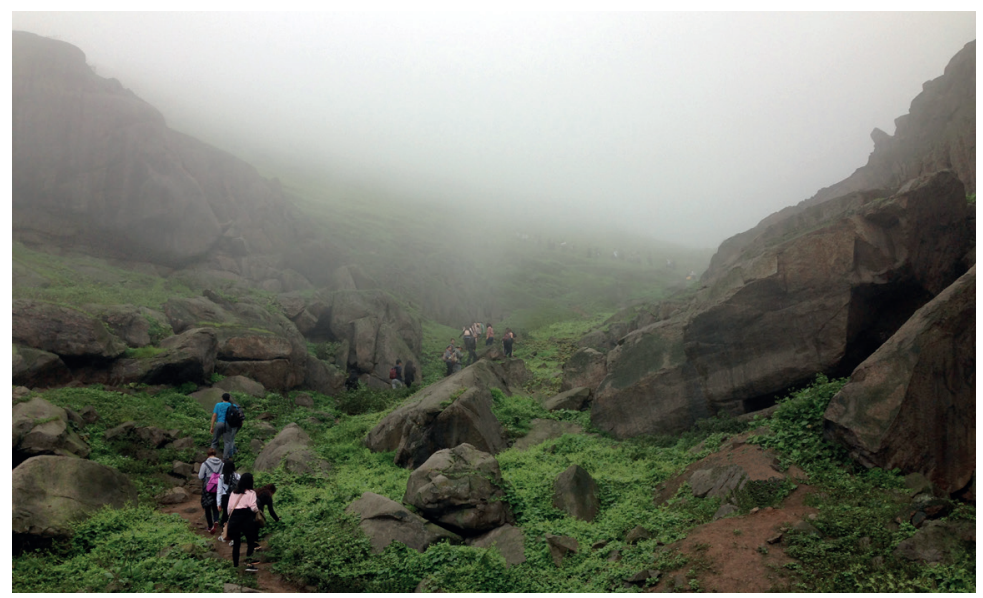

Figura 3.

Vista del camino a las lomas en temporada de niebla

Archivo fotográfico de los autores

Es relevante mencionar que las lomas de Amancaes poseen una fuerte carga histórica, debido a la fiesta de San Juan, evento que se desarrolló desde hace aproximadamente cuatrocientos años. Leguía (2007) señala que la celebración era un gran festival que tuvo lugar en la pampa de Amancaes, acompañado de manjares gastronómicos, música y danza, donde confluían las diferentes clases sociales. Es así como Amancaes reflejó los cambios sociales y culturales que acontecían en Lima en el siglo xx.

Sin embargo, el panorama actual revela que la mayor incidencia de pobreza del Rímac se encuentra precisamente en este sector, con índices de hasta 40,4 \% (Instituto Nacional de Estadística e Informática, 2013). A esto se le suman las alarmantes tasas de insalubridad. La jurisdicción del Rímac presenta un índice de dengue de 3,6\%, que es seriamente alto; y uno de los puntos más críticos es el asentamiento humano Flor de Amancaes. Según la Dirección de Redes Integradas de Salud Lima Norte (DIRIS, 2019), la tasa de prevalencia e incidencia de tuberculosis asciende a 184 casos por cada 100000 habitantes, posicionándose como la más elevada de Lima Norte; asimismo, los dos casos registrados de zika, reportados en el 2017, se presentaron en este lugar (Comité Distrital de Seguridad Ciudadana del Rímac, 2017).

Este barrio sigue los patrones recurrentes de las invasiones en periferia, con una falta de preocupación por el ordenamiento territorial donde se hace 
notoria la ausencia de espacios públicos, equipamiento educativo, de salud y seguridad. Paralelamente, las lomas de Amancaes se encuentran, hoy en día, atravesando su mayor reto, que es el de subsistir frente a una fuerte presión urbana que no parece tener intenciones de detenerse. Con base en un acercamiento al lugar, se evidencia la desaparición del concepto de lomas en el imaginario colectivo de la población; y, además, se percibe que la memoria del lugar está relacionada con los fuertes vínculos de la comunidad.

Con base en lo expuesto, urge la presencia de una intervención que regule el conflicto entre el ambiente natural y el urbano, bajo un proyecto emplazado en el borde, debido a que este último:

Se personifica en un espacio particular, identificado y apropiado por los actores que participan en su propia producción en la medida en que afecta las estrategias y accionar de los agentes, induciéndolos a cuestionar su identidad, su relación con el entorno y sus apuestas para este. (BallénVelásquez, 2014, p. 33)

Esto quiere decir que las estrategias planteadas en el borde representarán un impacto contundente en el ordenamiento territorial (morfología) y la interacción sociocultural que este generará, de modo tal que fortalezcan los lazos entre las lomas y la comunidad. Es necesario, además, que el proyecto se sensibilice con las necesidades que demanda la población, para mejorar la calidad de vida. Por eso, se requiere diversificar su radio de acción a través de núcleos funcionales, ubicados estratégicamente para que respondan a las necesidades ecológicas, de accesibilidad y equipamiento.

Es por ello que retomamos la idea inicial de percepción del lugar y la procesamos hacia un enfoque del rol arquitectónico frente a la problemática mencionada. En estos términos, se plantea una intervención con base en la participación ciudadana que va a componer estrategias de diseño centrado en el poblador, para que este genere la construcción social del territorio. Esto trae como producto la reconstrucción de la memoria colectiva, el restablecimiento de valores, el fortalecimiento de la autonomía y el enriquecimiento de la identidad de la población (Aguilera-Martínez et al., 2017).

En esta línea de trabajo se propone un proyecto de borde que, en primera instancia, salvaguarde las lomas y, a su vez, sea sensible a las necesidades urgentes de la población; por ello, se propone un reordenamiento territorial y diversificación funcional del borde. En segundo lugar, ayudará a contener el crecimiento urbano por medio del respeto al entorno natural incorporado en el imaginario colectivo de la comunidad. Adicionalmente, incentivará el entendimiento del paisaje como un nuevo recurso económico ligado a la multifuncionalidad y una potente corriente turística basada en el potencial de ese paisaje y de su patrimonio cultural. 
TOMA DE PARTIDO

El proyecto se concibe como un ecotono ${ }^{2}$, es decir, su naturaleza es el resultado de la transición entre entornos contrastantes: las lomas y la trama urbana. No se establecen límites claros, lo que permite dejar de lado las dicotomías para entenderlo como una nueva entidad que surge, a partir de ambas realidades, y las vincula. Con base en un entendimiento geométrico del contexto, la intervención debe ser una prótesis para la loma, que busque regenerar el perfil natural perdido; por lo tanto, el proyecto no se mimetiza, camufla ni sobrepone al terreno.

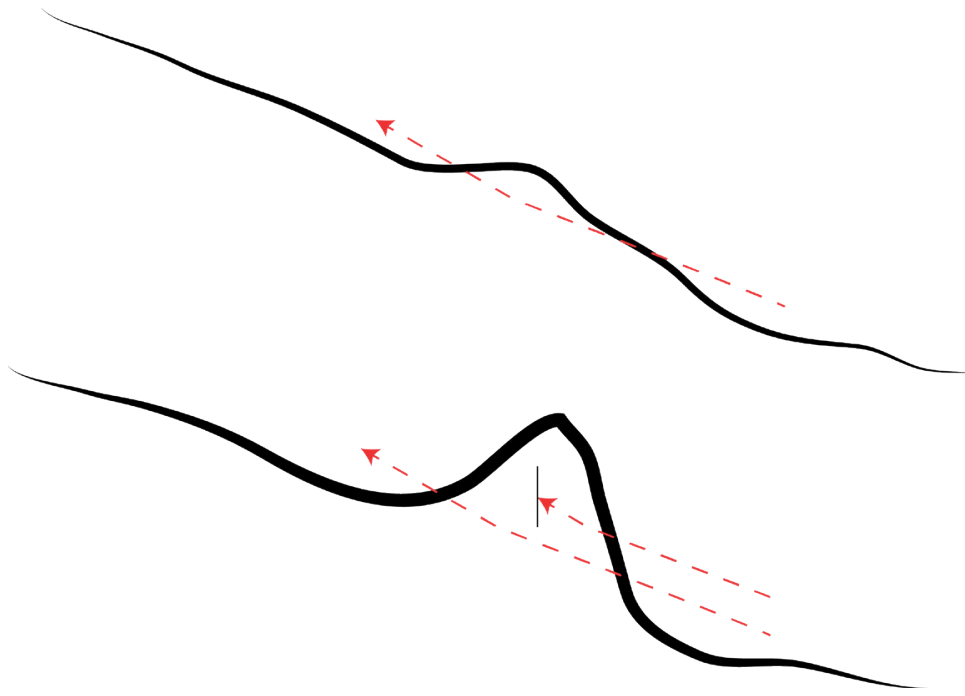

A lo largo de su recorrido se experimentan diversas alteraciones, producto de su adaptación a dos medios en conflicto, donde del choque de la trama urbana y la naturaleza de la loma surge una masa configurada con base en el ritmo de ambas; por ello, se comporta como un trayecto irregular, que, de acuerdo a lo que el contexto requiera, permite momentos de transición o freno.

2 El ecotono es definido por Clements (1905) como una intersección entre dos ecosistemas distintos, donde los procesos de intercambio son evidentes. El efecto del ecotono implica una mayor riqueza en el área con respecto a los ambientes adyacentes, el cual puede definirse como un nuevo hábitat diferente. El ecotono urbano, según Cuesta Beleño (2012), hace referencia a territorios relacionados con lo emergente o espacios de transición en diferentes escalas reconocidos por su potencial de cohesión y adherencia hacia otros territorios, y por su conformación sociocultural; dichos ecotonos urbanos se encuentran constituidos por elementos urbanos, ambientales o socioculturales que provienen de las estructuras urbanas contiguas, por lo que expresan un carácter dual de dependencia e independencia.
Figura 4.

Comportamiento de la figura como ecotono

Elaboración propia 
Figura 5

Comportamiento del proyecto a modo de prótesis de la loma

Elaboración propia

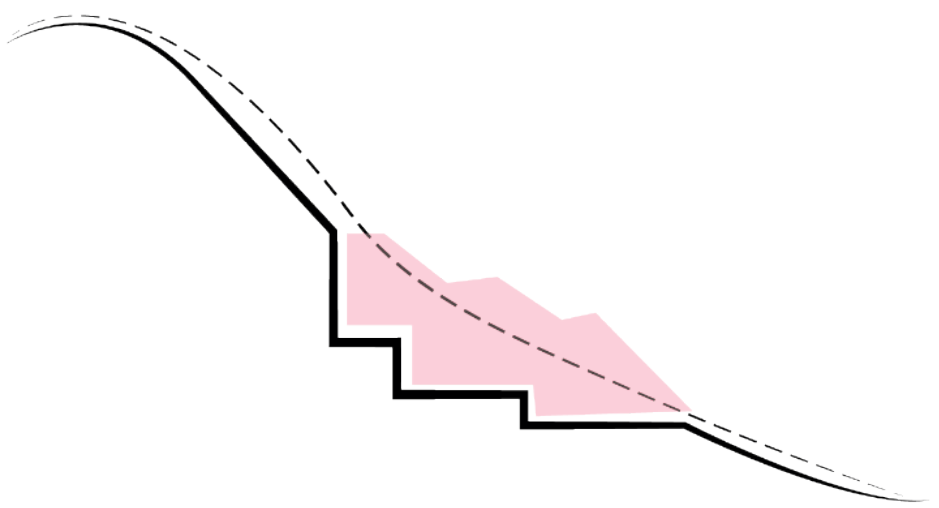

Finalmente, se busca que el proyecto de borde, como ecotono, logre superar el enfrentamiento rural-urbano, se adapte y no sea reducido a un límite estático, sino que frene las invasiones y salvaguarde la loma.

\section{MASTER PLAN}

Como primer paso para resolver este enfrentamiento, encontramos la necesidad de proponer un plan integral urbano a lo largo del borde de la loma para reestructurar una nueva dirección en la dinámica loma-ciudad y componer, mediante intervenciones paisajísticas, el espacio público e infraestructura. El plan consiste en articular el borde entre la ciudad y la loma mediante núcleos programáticos. Se emplazan una serie de edificios, a modo de núcleos, que presentan un programa estratégico respecto a su ubicación, articulados entre ellos por una alameda que se desarrolla en toda la extensión del borde y genera la activación del espacio público ubicado entre las conexiones.

La constitución del programa de cada núcleo surge a partir de las necesidades primordiales de la loma, su preservación y la mejora de la calidad de vida de la población en el ámbito económico, social y natural, donde se tiene como variable principal el gran problema de accesibilidad. Por lo tanto, se proponen distintos bloques programáticos enfocados tanto en la población como en la loma, los cuales se clasifican en seis funciones: salud, social, educativa, productiva, cultural y medioambiental. Se descentralizan las funciones, sin perder su calidad de híbrido, de tal menara que cada núcleo tiene una cabeza funcional, seleccionada con base en las carencias del lugar. Estas son las siguientes: centro de salud, auditorio, biblioteca central, mercado, centro cultural y centro de interpretación; cada uno va acompañado de un programa complementario como salones comunales, talleres educativos y servicios, entre otros. De esta manera, los usuarios pueden realizar diversas actividades en el mismo núcleo y activar el espacio público. 
Según el análisis de "escalas humanas y conexión en tramos" de la teoría de "red urbana" por Salingaros (2005), la trayectoria no debe proponerse únicamente como caminos, sino que debe contener subnúcleos para incentivar la continuidad de dicho recorrido y mantener su funcionalidad. Es por ello que se propone una serie de puntos de comercio y servicios, a distancias intermedias que terminan de articular toda la red. Dicha intervención va de la mano con un tratamiento paisajístico que abarque desde la reforestación de la loma, la propuesta de sembríos hasta la introducción de especies que mantengan el paisaje verde todo el año.

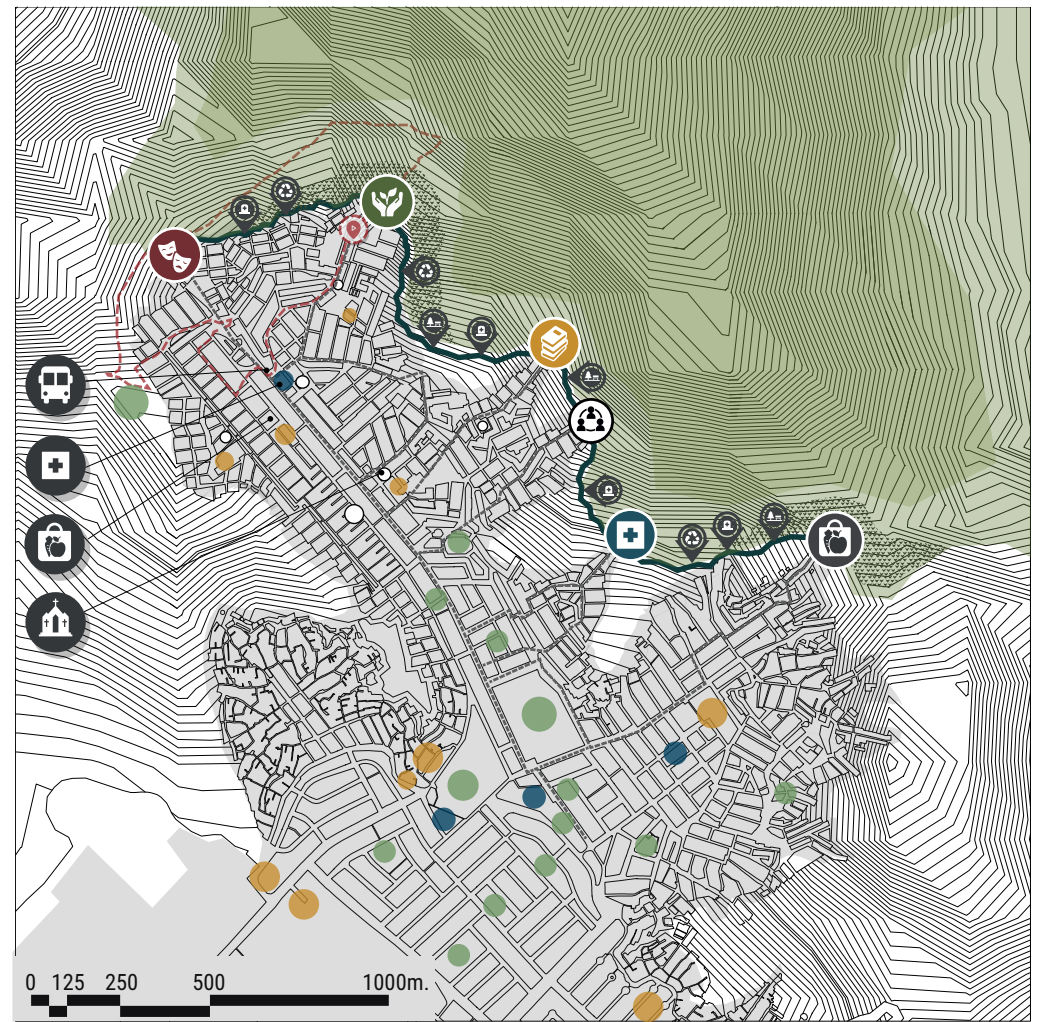

\section{ESTRATEGIAS PROYECTUALES}

Como el proyecto presenta dos escalas distintas, se plantea un master plan a nivel macro. Este articula el borde entre la loma de Amancaes y los asentamientos que la colindan; y, a nivel micro, seis núcleos de activación barrial dentro de la intervención. Es por ello que, de la mano de la toma de partido, establecemos una serie de lineamientos, a modo de estrategias

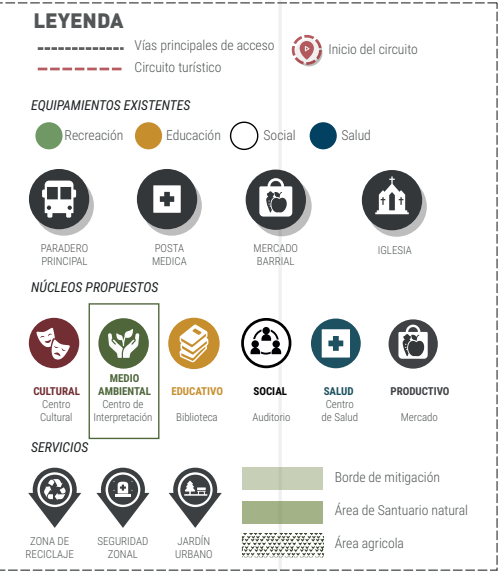

Figura 6.

Master plan

1

Elaboración propia 
Figura 7

Abstracción de límites desdibujados

Elaboración propia

Figura 8.

Peñascos más trama urbana resulta una nueva naturaleza

Elaboración propia proyectuales, que funcionen para ambas escalas, donde el principal concepto por desarrollar es el de proyecto como ecotono.

\section{Límites desdibujados}

El inicio y fin del edificio no están marcados en la intervención, de tal manera que no se entiende como un elemento aislado de la composición, sino como una continuidad del paisaje.

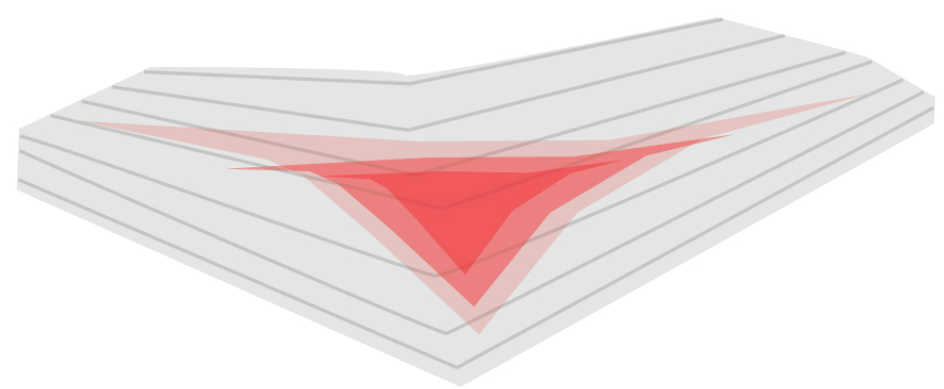

\section{Naturaleza ecléctica}

Tomar elementos de ambas condiciones para formar un carácter propio. Por lo tanto, se asimila la geometría de los peñascos, mediante el uso de la plegadura para acoplarse a la irregularidad de la superficie.

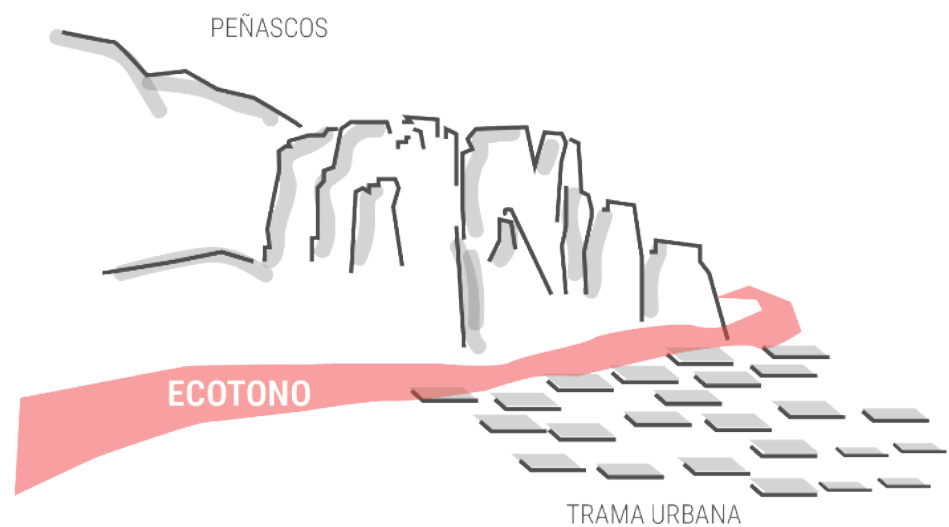




\section{Connotación de la escala}

El entendimiento del proyecto varía de acuerdo a la proximidad del usuario, con base en la paradoja de Mandelbrot $(1967)^{3}$, donde se evidencia un cambio de medida que depende de la escala. Extrapolamos esta idea al proyecto, de tal manera que, a una escala macro, la figura se entiende como un sólido continuo con una fuerte presencia que se acopla al paisaje junto a elementos como neblina y verdor; sin embargo, a una escala micro, el edificio es penetrable en su interior y se abstrae del contexto.

\section{CONNOTACIÓN DE LA ESCALA}

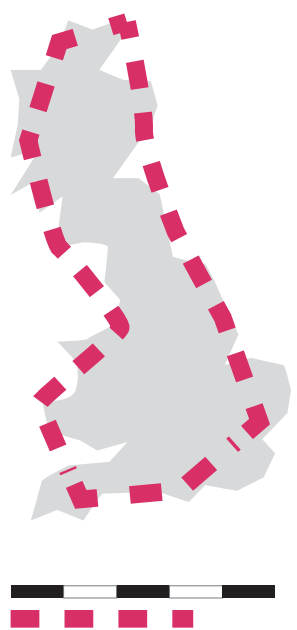

MACRO

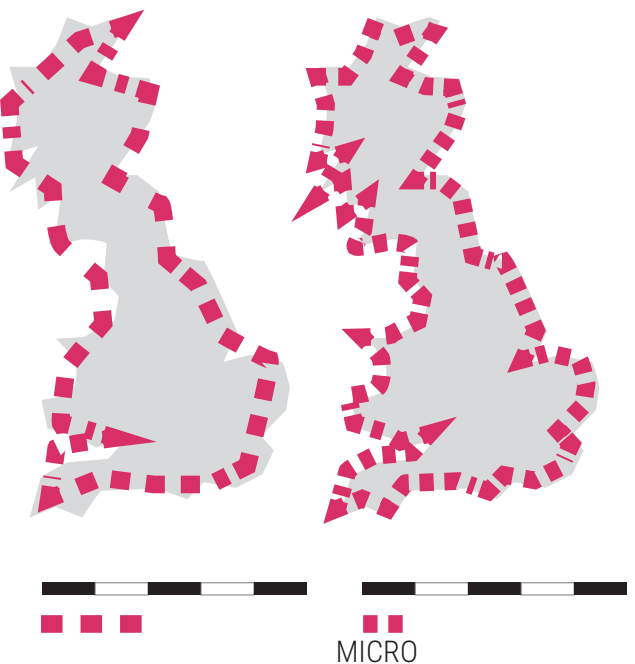

MICRO
Figura 9.

Extrapolación

de la paradoja al

proyecto de borde

Elaboración propia

\section{Cualidad de prótesis}

El volumen toca el suelo en su totalidad y se entiende como un elemento que pertenece al contexto, no se identifica como un intruso en el paisaje o un objeto sobrepuesto. Por lo tanto, la figura ayuda a completar la superficie dañada y manipulada por las invasiones a modo de prótesis.

3 La paradoja surge ante la interrogante de Mandelbrot (1967) respecto a la longitud de la línea costera de Gran Bretaña, la cual —señala - depende de la escala de medida: mientras esta se hace más pequeña, la longitud del litoral costero crece sin límites. Es decir, el entendimiento de una unidad varía de acuerdo a la relación de proximidad del observador. 
Figura 10

Comportamiento de la figura como prótesis

Elaboración propia

Figura 11 Permeabilidad de la membrana

Elaboración propia

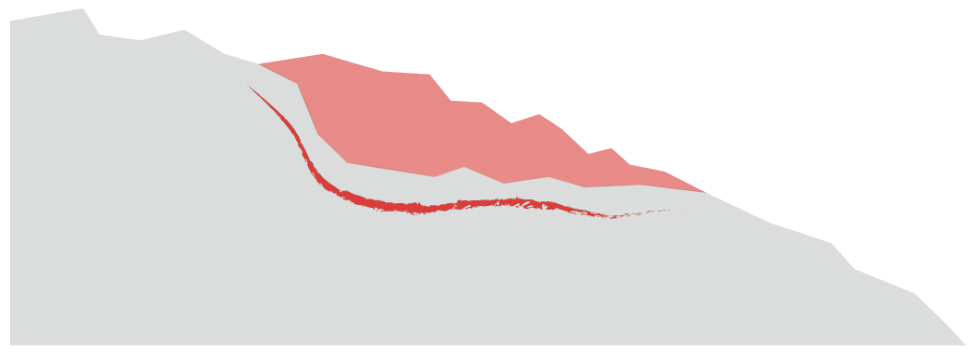

\section{Superficie ambivalente}

La permeabilidad de la superficie reacciona ante los tipos de flujo: en momentos en que necesita ser accesible para el usuario, funciona como una membrana que abre camino, mientras que en momentos en que la loma requiera mayor privacidad para su conservación, esta se rigidiza e impide el paso; sin embargo, en ambos momentos, el edificio sigue entendiéndose como una unidad.

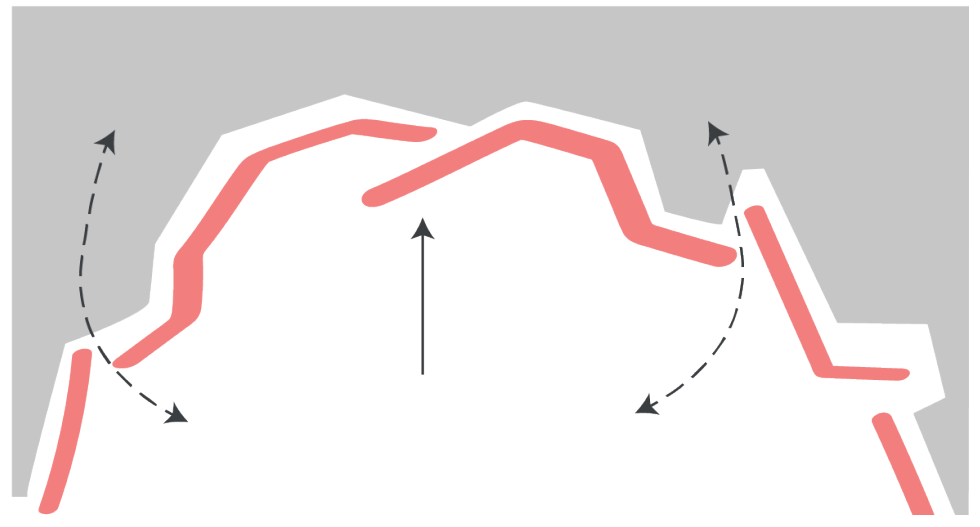

\section{Sistematización}

$\mathrm{Al}$ ser un proyecto de carácter longitudinal, se sistematizó el funcionamiento en cuanto a las activaciones del lugar, jerarquías del programa, zonificación y tipos de uso. El trazo de la composición se encuentra basado en la curva de nivel de la loma. El resultado es un recorrido irregular, que funciona como línea base para la ramificación del programa alrededor de este. 


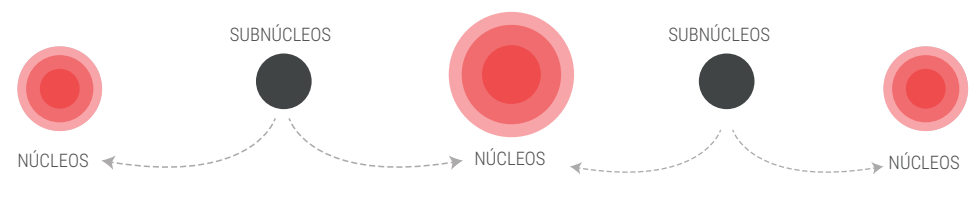

Figura 12.

Sistematización del programa

Elaboración propia

\section{Análisis del usuario}

Con base en una población de 43696 habitantes, según la proyección al 2020 (Mientorno.pe, 2020), se realizó el diagnóstico de los usuarios. Se vio por conveniente dividirlos en dos categorías: el poblador local, que a su vez se divide en niños y adultos; y el usuario externo, que se refiere a los visitantes o turistas. Ambos tipos detallan una serie de necesidades insatisfechas como estas:

a. Poblador local (adulto y niño)

- Presentan estudios incompletos.

- Trabajan de manera informal y a temprana edad.

- Son fervientes en su fe.

- Pertenecen al sector socioeconómico D.

- Desconocen la importancia de la loma y no tienen acceso a educación ambiental.

- Se abastecen en lugares alejados a sus puntos de venta.

- Presentan problemas de salud a temprana edad (desnutrición, anemia, tuberculosis, neumonía).

- Son muy propensos a enfermedades infecciosas por falta de higiene.

- $\quad$ Tienen un fuerte sentido de comunidad.

- No cuentan con espacios lúdicos seguros ni de esparcimiento dentro o fuera de casa.

b. Usuario externo (visitante)

- Desconoce la historia de la loma.

- Busca conexión con la naturaleza y una experiencia diferente a la ciudad.

- Requiere espacios de permanencia al aire libre.

- Participa de deportes de aventura.

- Valora el paisaje y su diversidad.

- Está dispuesto a invertir en su visita.

Del mismo modo que se descentralizan las funciones en el master plan con cinco núcleos interdependientes, se realiza la misma analogía con 
cada núcleo y su programa, de manera que sus funciones se encuentren repartidas en zonas. Como resultado de dicha analogía y del diagnóstico de los usuarios, se han enumerado cinco metas para contrarrestar las carencias existentes, asignándole un propósito a cada zona del programa.

- Meta 1: facilitarles el acceso a recursos educativos, impulsar la educación ambiental y generar conciencia sobre el patrimonio natural de Amancaes. Es por ello que el proyecto debe tener espacios enfocados en estas necesidades mediante una zona de educación.

- Meta 2: conocer la carga histórico-simbólica del lugar y su ecosistema tanto para usuarios locales como externos. Asimismo, lograr que el usuario se sienta parte del ecosistema al recorrer la atmósfera del proyecto, de tal manera que el usuario se sienta partícipe. Por lo tanto, se debe contar con una zona de interpretación.

- Meta 3: fomentar el desarrollo de actividades, de tal manera que la población genere sus propios ingresos y continúe manteniendo el equilibrio dentro del ecosistema. Por ello, es indispensable la presencia de una zona de producción.

- Meta 4: brindar espacios públicos de calidad para el desarrollo social de la comunidad, áreas de esparcimiento que se encuentren ligadas a la naturaleza, así como una mejor alternativa para el desarrollo de actividades comunitarias. Por tal motivo, se presenta la necesidad de una zona social.

- Meta 5: rescatar el fuerte fervor espiritual de la comunidad, que genera unión e importantes lazos dentro de la población local. Por ello, es imprescindible la presencia de una zona de culto.

\section{PROGRAMA}

En este artículo, el núcleo por desarrollar, dentro del proyecto de borde, es el centro de interpretación, ubicado en la entrada principal al camino a la loma; asimismo, se encuentra conformado por cinco zonas principales clasificadas por sus funciones: zona de educación, zona de preservación, zona de producción, zona social y zona de culto, cada una ligada a una zona de administración y servicios. Así también, entre zonas, existen espacios complementarios como talleres de capacitación y servicios. Estas zonas se encuentran conectadas por los espacios de exposición, salas de interpretación y muestras temporales. Del mismo modo, en la parte superior, existe otro tipo de programa ligado a la naturaleza y al espacio público, como plazas que se activan a través de las zonas principales, zonas de estadía, miradores, huertos y jardín botánico. 


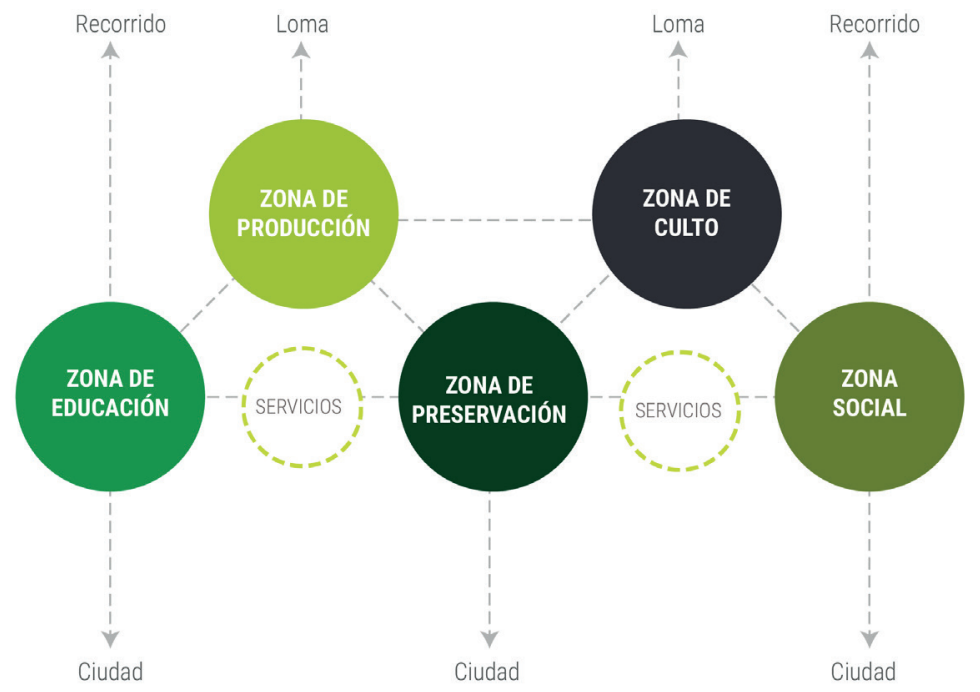

Figura 13

Programa

del centro de

interpretación

Elaboración propia

\section{SOSTENIBILIDAD DEL PROYECTO}

Al encontrarnos en un ecosistema tan frágil como lo es la loma, que se ha visto degradado con el transcurso del tiempo, fuimos muy cuidadosos al plantear una intervención (véase la figura 4) de esta naturaleza. Es por ello que, mediante lo antes explicado, no solo se recupera su riqueza natural, sino que la intervención no es invasiva y hace buen uso de los recursos naturales de la zona sin explotarlos, en beneficio de la población y la loma. 
Figura 14

Bosquejo de una sección transversal

Elaboración propia

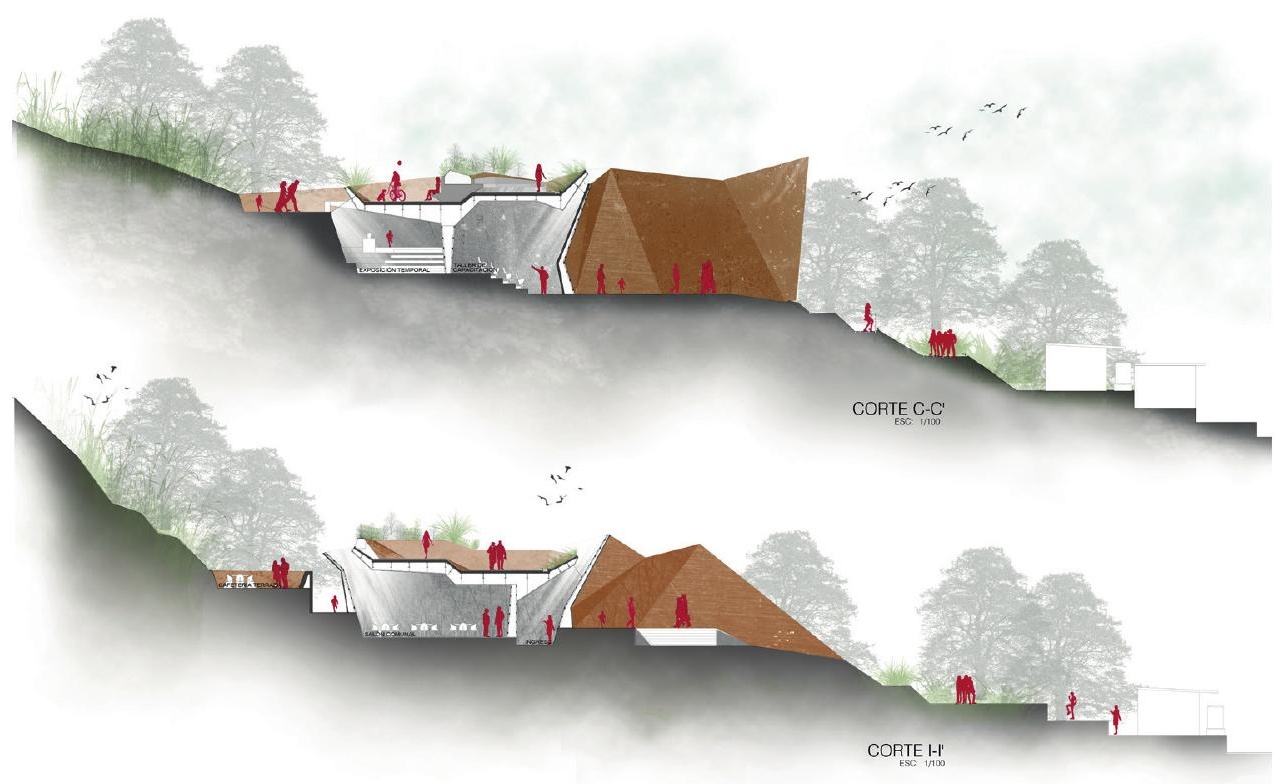

El factor humedad puede sonar perjudicial para cualquier proyecto; sin embargo, fue convertido en la principal fortaleza, de manera que obtiene un mayor protagonismo al adaptarse a ella. El proyecto cuenta con espacios que permiten el paso de la neblina mediante aberturas, que logran la participación activa en la percepción del usuario. Del mismo modo, su recolección, por medio de atrapanieblas, contribuye al suministro de agua, y no como un elemento aislado, sino que se integra a la arquitectura, a modo de barandas, mobiliario y superficies, lo cual al mismo tiempo favorece la proliferación de la vegetación que se utiliza en la fachada del proyecto.

Con respecto al factor pendiente, el proyecto se desarrolla en paralelo a la curva de nivel y no transversal, debido a que el ir contra la pendiente condiciona al volumen a enterrarse y ser más invasivo con el terreno. Con base en esta estrategia, se contienen espacios más longitudinales y flexibles que permiten una interrelación espacial entre programas independientes a desnivel, al aprovechar la pendiente como parte del desarrollo arquitectónico. Asimismo, en un entorno de ladera, es valioso contar con un plano horizontal que permita el desarrollo social de la población; por ello, todo el proyecto de borde se encuentra integrado por una alameda que activa el espacio público, lo que genera una mayor seguridad en la zona.

Paralelamente, se aprovecha el factor agrícola del entorno como uno de los pocos puntos verdes dentro de la ciudad, en el que se fomenta el desarrollo agrícola y productivo de la población al contribuir a su seguridad alimentaria; es decir que se tenga acceso a un suministro suficiente de alimento para un abastecimiento local. En consecuencia, se tiene un asentamiento concientizado 
con respecto al valor natural que la loma provee, como resultado de la agricultura local y promoción directa de los productos.

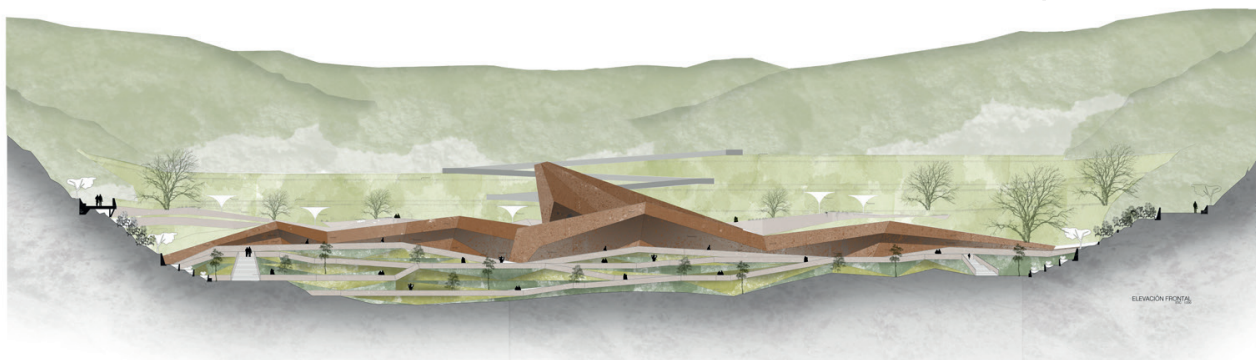

\section{DESARROLLO PAISAJÍSTICO}

El reto principal fue integrar todas estas intenciones en la reconstrucción de un paisaje dañado, mediante la reorganización de la geometría natural como extensión del edificio, donde se percibe la continuidad entre el paisaje generado y el borde. Asimismo, una de las motivaciones fue crear un espacio previo al inicio del recorrido hacia las lomas, de tal manera que todo el edificio puede ser visto como una serie de espacios preliminares que conducen e incentivan a recorrer las lomas de Amancaes.

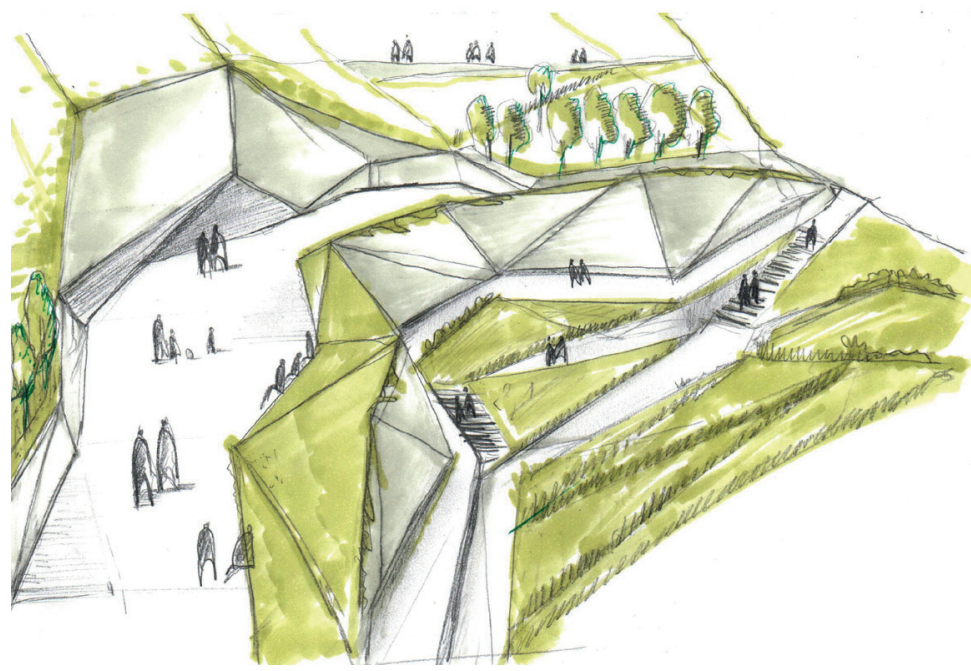

Existe una constante relación proyecto-naturaleza, que se hace notoria desde el recorrido principal, que mantiene, a lo largo, una conexión directa con la loma. Aquí se desarrolló un diseño paisajístico con las especies del lugar, emplazadas en andenerías que mantienen la lógica compositiva del edificio; estas contienen sembríos para reforestación y abastecimiento, espacios de
Bosquejo de la intervención

Elaboración propia

Figura 16

Vista del proyecto desde el recorrido principal

Elaboración propia 
Figura 17

Sistematización de

programa

Elaboración propia mirador y atrapanieblas. Por otro lado, el recorrido se ve acompañado por una franja verde que, como parte del edificio, se adapta a los cambios espaciales y de nivel; en algunos momentos se comporta como baranda, en otros se levanta para conformar espacios de estadía, además de contrastar las superficies de concreto.

Quisimos romper la tensión entre las viviendas y el inicio del edificio al generar un espacio de transición que no le pertenezca a ninguno, pero que funcione para ambos, de tal manera que signifique un precedente al edificio y una extensión del espacio público para la ciudad. Este inicia con una serie de rampas de ingreso en el remate de las vías principales de la zona, las cuales van acompañadas de un tratamiento de superficies, a modo de andenería hecha de pircas, y un ligero colchón verde que controla la visual en ambos sentidos; dichas rampas conducen a plazas intermedias que se conectan con el zócalo que circunda los ingresos principales del edificio. El tratamiento desarrollado permite incorporar el concepto de naturaleza a lo largo del borde y vincularla directamente con la población.

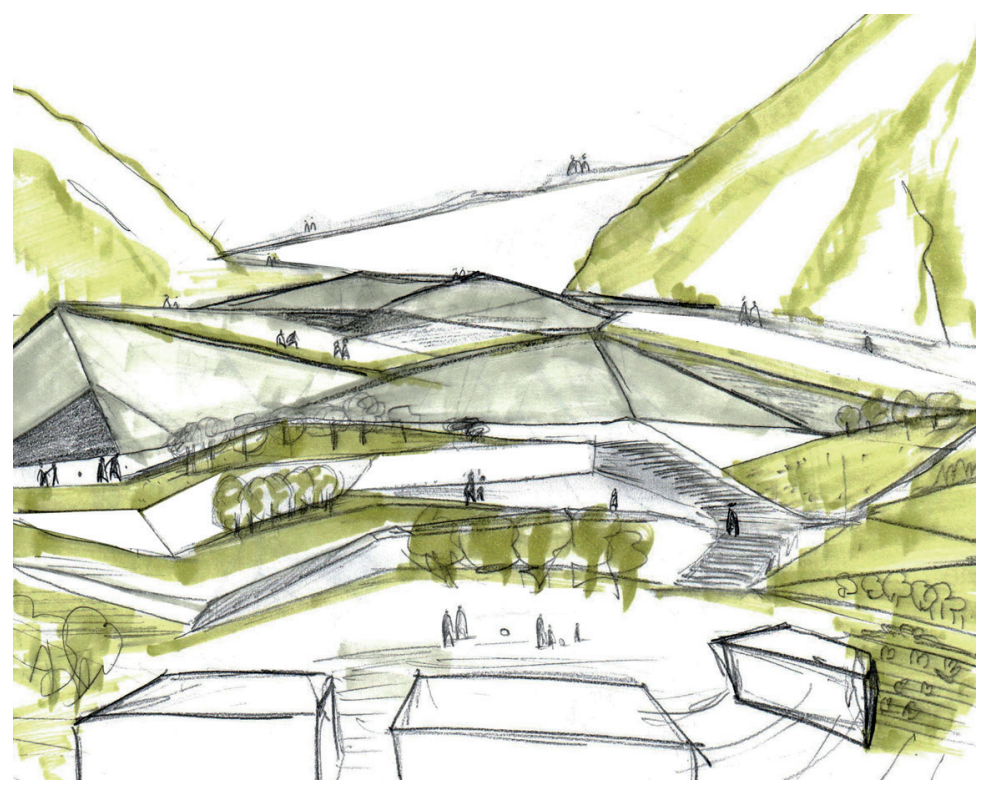

El criterio de composición es producto de la adición de diferentes componentes que comienza en el estrato urbano, atraviesa una serie de capas de vegetación silvestre, superficies duras de circulación y estadía, espacios programáticos del edificio, vegetación en peligro, huertos y sembríos, y, finalmente, la misma loma. Todo esto nos permite referirnos al proyecto como un ecotono; esto es, se ha logrado conformar un espacio de transición con carácter propio, reflejado en su composición geométrica de triangulación que termina por enriquecer a ambas partes. 


\section{DISCUSIÓN}

En la actualidad existe un conflicto entre el entorno natural de la loma de Amancaes y el ambiente construido adyacente sin adecuada planificación y constante expansión. Esto ha generado, en primer lugar, un fuerte impacto sobre el ambiente, donde el ecosistema se encuentra en la fase más vulnerable, pues su acelerada degradación conduciría a su posible pérdida.

En segundo lugar, la población ha ido creciendo en desmedro del ambiente natural y su desarrollo ha generado que ellos mismos sean parte de los principales perjudicados. Al habitar en un ambiente improvisado, no se tiene como prioridad la incorporación de equipamiento urbano que satisfaga sus necesidades a futuro, por lo que una vez asentados se hace evidente la ausencia de dicha infraestructura.

Sin embargo, la población da la espalda a la enorme diversidad biológica y a los posibles recursos naturales que pueden ser aprovechados, por lo que se ven degradados por la antropización, lo que, sumado a la evidente falta de ingresos, conduce a una precaria calidad de vida.

Nuestro rol ha sido el de afrontar las diferentes variables del lugar, sumadas a la particularidad de intervenir un borde para lograr el equilibrio entre la preservación del ecosistema y la contribución a favor del desarrollo de la población. Si bien la intención del proyecto es solucionar el conflicto actual mediante la intervención espacial, es el efecto generado lo que va a trascender en el tiempo y en la dinámica social para los futuros habitantes de Amancaes y Lima Metropolitana.

\section{REFERENCIAS}

Aguilera-Martínez, F. A., Medina-Ruiz, M., Castellanos-Escobar, M. C., y PerillaAgudelo, K. J. (2017). Intervención social en el borde urbano desde el proceso de la significación cultural. Revista de Arquitectura, 19(2), 78-93.

Ballén-Velásquez, L. M. (2014). "Desbordando" la categoría de borde. Reflexiones desde la experiencia bogotana. Bitácora Urbano-Territorial, 2(24), 31-41.

Caballero Zeitún, E. L. (2011). El concepto de ladera urbana. Ciencias Espaciales, 4(1), 41-61.

Clements, F. E. (1905). Research Methods in Ecology. University Publishing Company.

Comité Distrital de Seguridad Ciudadana del Rímac. (2017). Plan local de seguridad ciudadana 2018 - Rímac. Municipalidad Distrital del Rímac. https:// docplayer.es/110370362-Plan-local-de-seguridad-ciudadana-rimac.html

Corell, D. (2014). Estudio estadístico de la potencialidad de uso del agua de niebla como recurso hídrico en el litoral mediterráneo de la Península Ibérica [Tesis doctoral; Universidad Politécnica de Valencia]. http://hdl.handle. net/10251/48523 
Cuesta Beleño, A. C. (2012). Ecotono urbano: introducción conceptual para la alternatividad al desarrollo urbano. Universidad de La Salle.

Del Castillo, J. M. (2017). Propuesta técnica para declarar de interés distrital el reconocimiento de los ecosistemas de "Lomas de Amancaes" como áreas de reserva ambiental del Rímac. Municipalidad del Rímac.

Dirección de Redes Integradas de Salud Lima Norte. (2019). Análisis de la situación de salud 2018. Ministerio de Salud. http://www.dirislimanorte.gob.pe/ wp-content/uploads/2019/01/asis-2018.pdf

Instituto Nacional de Estadística e Informática. (2013). Planos estratificados de Lima Metropolitana a nivel de manzana 2016. Según ingreso per cápita del hogar. Según grupos de pobreza monetaria. https://www.inei.gob.pe/ media/MenuRecursivo/publicaciones_digitales/Est/Lib1403/index.htm

Leguía, E. (2007). Lima 1919-1930, la Lima de Leguía. Universidad Nacional Mayor de San Marcos, Fondo Editorial.

Lynch, K. (1959). La imagen de la ciudad. Editorial Infinito.

Mandelbrot, B. (1967). How Long is the Coast of Britain? Statistical SelfSimilarity and Fractional Dimension. Science, 156(3775), 636-638.

Mientorno.pe (2020). Flor de Amancaes, Cercado de Lima, Perú. INEI. https://www. mientorno.pe/informe/flordeamancaes

Municipalidad de Lima. (2019). Expediente técnico. Propuesta de Área de Conservación Regional Sistema de Lomas de Lima. http://pgrlm.gob.pe/ wp-content/uploads/sites/30/2019/10/Sistema_de_Lomas.pdf

Nieuwland, B., y Mamani, J. M., (2017). Las lomas de Lima: enfocando ecosistemas desérticos como espacios abiertos en Lima metropolitana. Espacio y Desarrollo, 29, 109-133.

Programa de las Naciones Unidas para el Desarrollo. (2018). Proyecto EbA Lomas. Retos y oportunidades de la conservación de las lomas de Lima Metropolitana. https://www.pe.undp.org/content/peru/es/home/library/eba-lomas.html

Salingaros, N. (2005). Teoría de la red urbana (N. F. Hernández Amador, Trad.). En Principles of Urban Structure. Design, Science Planning (pp. 15-38). Techne Press. https://repositorio.tec.mx/bitstream/handle/11285/573456/ DocsTec_11417.pdf?sequence=1\&isAllowed=y\#page=6“

Servicio Nacional de Áreas Naturales Protegidas. (2013). Gestión del agua y biodiversidad en la Reserva Nacional de Lachay. Ministerio del Ambiente. https://www.minam.gob.pe/cambioclimatico/wp-content/uploads/sites /11/2015/01/Gestión-del-Agua-y-Biodiversidad-en-la-Reserva-NacionalDe-Lachay.docx.pdf

Toro Vasco, C. (2005). Los servicios públicos y su relación con la expansión urbana en zonas de borde. Revista Ingenierías Universidad de Medellín, 4(6), 98-107. 